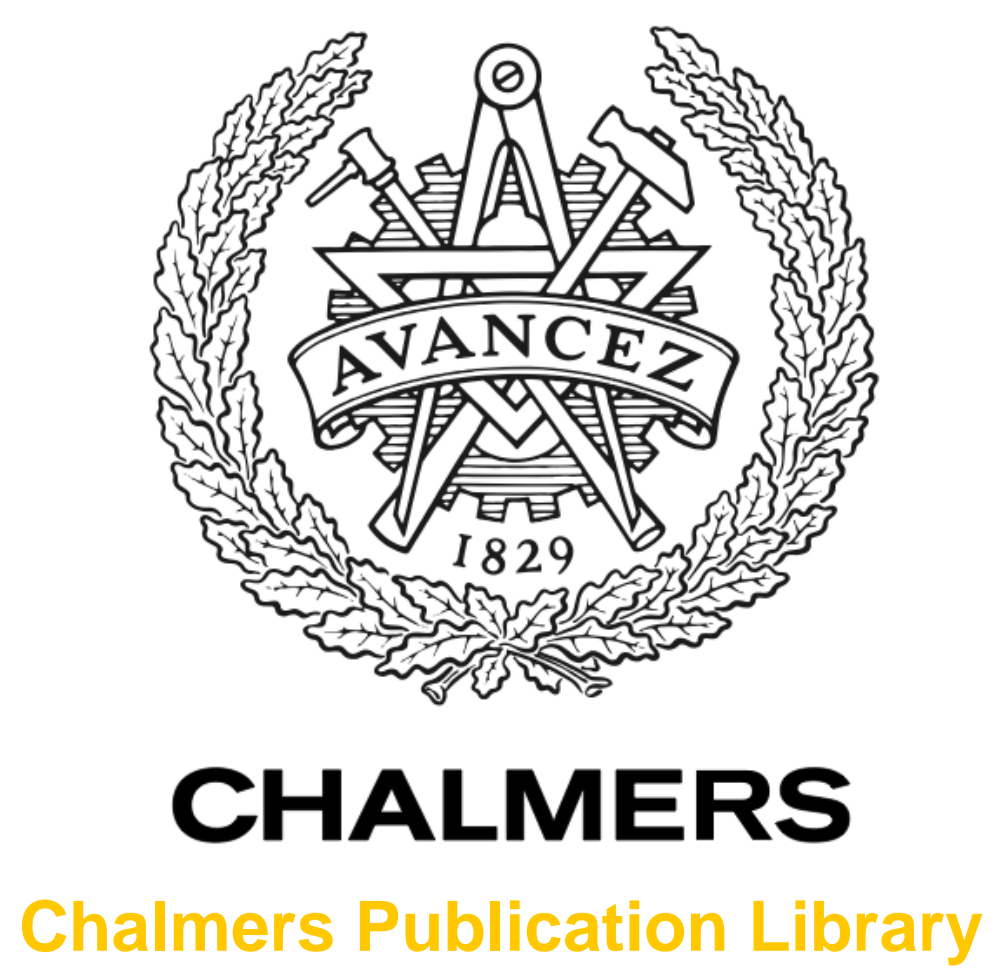

Multi-port matching efficiency in antenna systems with cascaded networks

This document has been downloaded from Chalmers Publication Library (CPL). It is the author's version of a work that was accepted for publication in:

15th International Symposium on Antenna Technology and Applied Electromagnetics, ANTEM 2012, Toulouse, 25-28 June 2012

Citation for the published paper:

Jamaly, N. ; Derneryd, A. (2012) "Multi-port matching efficiency in antenna systems with cascaded networks". 15th International Symposium on Antenna Technology and Applied Electromagnetics, ANTEM 2012, Toulouse, 25-28 June 2012

http://dx.doi.org/10.1109/ANTEM.2012.6262327

Downloaded from: http://publications.lib.chalmers.se/publication/164216

Notice: Changes introduced as a result of publishing processes such as copy-editing and formatting may not be reflected in this document. For a definitive version of this work, please refer to the published source. Please note that access to the published version might require a subscription.

Chalmers Publication Library (CPL) offers the possibility of retrieving research publications produced at Chalmers University of Technology. It covers all types of publications: articles, dissertations, licentiate theses, masters theses, conference papers, reports etc. Since 2006 it is the official tool for Chalmers official publication statistics. To ensure that Chalmers research results are disseminated as widely as possible, an Open Access Policy has been adopted.

The CPL service is administrated and maintained by Chalmers Library. 


\section{Multi-port Matching Efficiency in Antenna Systems with Cascaded Networks}

\author{
Nima Jamaly \\ Chalmers University of Technology \\ Gothenburg, Sweden 41296 \\ Email: jamaly@chalmers.se
}

\author{
Anders Derneryd \\ Ericsson Research, Ericsson AB \\ Gothenburg, Sweden 41756 \\ Email: anders.derneryd@ericsson.com
}

\begin{abstract}
A general compact formula for multi-port matching efficiency in the presence of an arbitrary number of cascaded networks is introduced. A comparison between the decoupling efficiency and multi-port matching efficiency is presented. Similarly, a compact formula for decoupling efficiency in a cascaded chain is provided. The presented compact formulas are quite advantageous for optimization of radiation efficiencies by designing a proper matching network.
\end{abstract}

\section{INTRODUCTION}

Among different radiation efficiency metrics for performance description of multi-port antennas, total active reflection coefficient (TARC) [1] , mean effective gain (MEG) [2], total embedded element efficiency [3], decoupling efficiency [4], and multi-port matching efficiency are commonplace [5]. Each metric has its own advantages and disadvantages. Nevertheless, the multi-port matching efficiency is handier to deal with for certain reasons. For instance, it depends solely on the input network parameters of the radiation system, e.g., S-parameters, which is easy to measure. Besides, in case of a lossless structure, it equates the corresponding total embedded element efficiency. Also under this lossless constraint, if the mean effective directivity is known, it can yield the corresponding MEG. Moreover, similar to TARC, it includes the excitation scheme as a degree of freedom in the radiation efficiency metric. To calculate multi-port matching efficiency, a compact formula has been introduced for an antenna system terminated by an arbitrary set of impedances [5]. Yet, in recent multi-port wireless systems, it is rare to find antennas connected directly to the receiver ports. In contrast, antennas are integrated to the receiver through a chain of cascaded networks. For example, dependent upon application and design, the cascaded networks can include a Butler network used for beam-forming, or different matching networks [6]. Hence, it is an advantage to be able to quickly calculate the multi-port matching efficiency in the presence of a number of cascaded networks. The latter establishes the main purpose of this paper, in which we derive a general compact formula for the aforementioned metric in a cascaded system.

The structure of this paper is as follows. In the second section, we derive a compact formula for a case of two cascaded networks. This is followed in section III by a brief discussion about how to extent it to a larger number of cascaded networks. Section IV is dedicated to decoupling efficiencies. In addition to a fair comparison between the decoupling efficiency and the multi-port matching efficiency, a general compact formula for the former is derived.

\section{CASE of Two CASCADED Networks}

The main goal in this section is to derive a compact formula for multi-port matching efficiency in the presence of two cascaded microwave networks. Yet, before anything, we need to set a consistent notation. In this paper, we use bold capital letters to denote matrices. Besides, letters with an over bar signify column vectors. The dagger sign stands for Hermitian transposition. I denotes an $(n \times n)$ identity matrix with $n$ being the number of ports in the system. The incident and reflected waves are normalized in a way that their square gives the average power.

The microwave circuit model of an antenna system with two cascaded networks is depicted in Fig. 1. This system is excited by a source of certain internal impedances given in the diagonal matrix of $\mathbf{Z}_{s}$. The further necessary parameters to be used in our derivation are shown in the figure. As defined in [5], the multi-port matching efficiency for an arbitrary excitation scheme is the ratio between the accepted power $P_{\text {acc }}$ and the maximum available power from the source(s), $P_{\text {avs }}$. It is known that the accepted power by any passive microwave system can be achieved through the incident and reflected waves at its ports, which are linked by the associated reflection matrix, $\boldsymbol{\Gamma}$. Deriving the reflection matrices at different points in the system is fairly straightforward. Referring to Fig. 1 and following a similar approach to the one used by D. Pozar [7, pp. 192], we obtain these matrices as

$$
\begin{aligned}
& \boldsymbol{\Gamma}_{s}=\left(\mathbf{Z}_{s}+\mathbf{Z}_{\circ}\right)^{-1}\left(\mathbf{Z}_{s}-\mathbf{Z}_{\circ}\right) \\
& \boldsymbol{\Gamma}_{1}=\mathbf{S}_{11}^{x}+\mathbf{S}_{12}^{x} \boldsymbol{\Gamma}_{3}\left(\mathbf{I}-\mathbf{S}_{22}^{x} \boldsymbol{\Gamma}_{3}\right)^{-1} \mathbf{S}_{21}^{x} \\
& \boldsymbol{\Gamma}_{2}=\mathbf{S}_{22}^{x}+\mathbf{S}_{21}^{x} \boldsymbol{\Gamma}_{s}\left(\mathbf{I}-\mathbf{S}_{11}^{x} \boldsymbol{\Gamma}_{s}\right)^{-1} \mathbf{S}_{12}^{x} \\
& \boldsymbol{\Gamma}_{3}=\mathbf{S}_{11}^{y}+\mathbf{S}_{12}^{y} \mathbf{S}_{a}\left(\mathbf{I}-\mathbf{S}_{22}^{y} \mathbf{S}_{a}\right)^{-1} \mathbf{S}_{21}^{y} \\
& \boldsymbol{\Gamma}_{4}=\mathbf{S}_{22}^{y}+\mathbf{S}_{21}^{y} \boldsymbol{\Gamma}_{2}\left(\mathbf{I}-\mathbf{S}_{11}^{y} \boldsymbol{\Gamma}_{2}\right)^{-1} \mathbf{S}_{12}^{y}
\end{aligned}
$$

In parallel, note that $\mathbf{S}_{\text {ant }}$, as defined in [8], is an $(n+2) \times(n+2)$ matrix being generally a function of angular direction. Among different submatrices building up $\mathbf{S}_{\text {ant }}$, only the input ports' $\mathbf{S}$-matrix, $\mathbf{S}_{\mathrm{a}(n \times n)}$, is independent of angular direction, and thus of particular interest to us. 


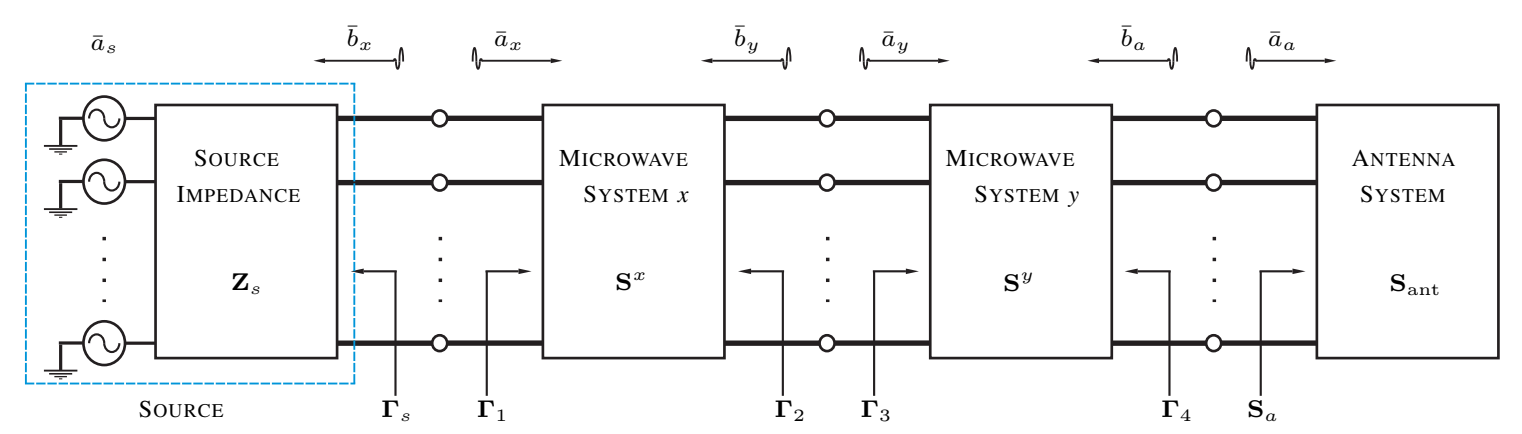

Fig. 1. Microwave circuit model of two cascaded networks with the multi-port antenna system.

\section{A. Formulation of Accepted Power}

A more challenging step towards our goal is to express the incident waves in terms of the known normalized voltage vector at the source, $\bar{a}_{s}$. Using voltage division rule in the fundamental circuit theory and later expressing the corresponding impedances with the associated reflection matrices, we arrive at

$$
\bar{a}_{x}=\left(\mathbf{I}+\boldsymbol{\Gamma}_{1}\right)^{-1} \mathbf{Q}^{-1} \bar{a}_{s}=\mathbf{T}_{s} \bar{a}_{s}
$$

where

$$
\mathbf{Q}=\left[\left(\mathbf{I}+\boldsymbol{\Gamma}_{s}\right)\left(\mathbf{I}-\boldsymbol{\Gamma}_{s}\right)^{-1}\left(\mathbf{I}-\boldsymbol{\Gamma}_{1}\right)\left(\mathbf{I}+\boldsymbol{\Gamma}_{1}\right)^{-1}+\mathbf{I}\right]
$$

and $\mathbf{T}_{s}$ is referred to as the source transmission matrix. For the input power to the first cascaded network, $P_{\text {acc }}^{x}$, we can write:

$$
P_{\mathrm{acc}}^{x}=\bar{a}_{s}^{\dagger} \mathbf{T}_{s}^{\dagger}\left(\mathbf{I}-\boldsymbol{\Gamma}_{1}^{\dagger} \boldsymbol{\Gamma}_{1}\right) \mathbf{T}_{s} \bar{a}_{s}
$$

On the other hand, following a similar path, we have

$$
\bar{a}_{y}=\left(\mathbf{I}-\mathbf{S}_{22}^{x} \boldsymbol{\Gamma}_{3}\right)^{-1} \mathbf{S}_{21}^{x} \bar{a}_{x}=\mathbf{T}_{x} \bar{a}_{x}
$$

with $\mathbf{T}_{x}$ being the transmission matrix of the first cascaded network. Accordingly, the accepted power at the second network, $P_{\text {acc }}^{y}$, becomes

$$
P_{\mathrm{acc}}^{y}=\bar{a}_{x}^{\dagger} \mathbf{T}_{x}^{\dagger}\left(\mathbf{I}-\boldsymbol{\Gamma}_{3}^{\dagger} \boldsymbol{\Gamma}_{3}\right) \mathbf{T}_{x} \bar{a}_{x}
$$

Furthermore, the incident wave at the antenna port can similarly be cast as follows:

$$
\bar{a}_{a}=\left(\mathbf{I}-\mathbf{S}_{22}^{y} \boldsymbol{\Gamma}_{4}\right)^{-1} \mathbf{S}_{21}^{y} \bar{a}_{y}=\mathbf{T}_{y} \bar{a}_{y}
$$

Using (2), (4) and (6), the total transmission matrix of the system which reads $\bar{a}_{a}=\mathbf{T}_{t} \bar{a}_{s}$ can be defined as

$$
\mathbf{T}_{t}=\mathbf{T}_{y} \mathbf{T}_{x} \mathbf{T}_{s}
$$

To calculate $\mathbf{T}_{t}$ one can first calculate $\boldsymbol{\Gamma}_{2} \& \boldsymbol{\Gamma}_{3}$, and accordingly $\boldsymbol{\Gamma}_{1} \& \boldsymbol{\Gamma}_{4}$ from (1). Then, by substituting them in (2), (4) and (6), the constituents of $\mathbf{T}_{t}$ in (7) are found.

Now, in a similar way as used in (3), we can achieve the accepted power by the multi-port antenna, $P_{\text {acc }}$, through

$$
P_{\text {acc }}=\bar{a}_{s}^{\dagger} \mathbf{T}_{t}^{\dagger}\left(\mathbf{I}-\mathbf{S}_{a}^{\dagger} \mathbf{S}_{a}\right) \mathbf{T}_{t} \bar{a}_{s}
$$

\section{B. Maximum Available Power from the Source}

A further step is to formulate the maximum available power from the source, $P_{\text {avs }}$. Recall that the maximum available power from the source can be transferred to the first cascaded system provided the equality $\boldsymbol{\Gamma}_{\mathbf{1}}=\boldsymbol{\Gamma}_{\mathrm{s}}{ }^{\dagger}$ holds. That means, $P_{\text {avs }}$ equates the accepted power by the first cascaded system, $P_{\text {acc }}^{x}$, under the aforementioned constraint.

$$
\begin{aligned}
P_{\mathrm{avs}} & =\left.P_{\mathrm{acc}}^{x}\right|_{\Gamma_{1}=\boldsymbol{\Gamma}_{\mathrm{s}}^{\dagger}} \\
& =\bar{a}_{s}^{\dagger} \mathbf{T}_{m}^{\dagger}\left(\mathbf{I}-\boldsymbol{\Gamma}_{s} \boldsymbol{\Gamma}_{s}^{\dagger}\right) \mathbf{T}_{m} \bar{a}_{s}
\end{aligned}
$$

in which

$$
\mathbf{T}_{m}=\left[\left(\mathbf{I}+\boldsymbol{\Gamma}_{s}\right)\left(\mathbf{I}-\boldsymbol{\Gamma}_{s}\right)^{-1}\left(\mathbf{I}-\boldsymbol{\Gamma}_{s}^{\dagger}\right)+\left(\mathbf{I}+\boldsymbol{\Gamma}_{s}^{\dagger}\right)\right]^{-1}
$$

Having all the required constituents in access enables us to formulate the multi-port matching efficiency, $e_{\mathrm{mp}}$, for any arbitrary excitation scheme, $\bar{a}_{s}$, as:

$$
\left.e_{\mathrm{mp}}\right|_{\bar{a}_{s}}=\frac{\bar{a}_{s}^{\dagger} \mathbf{T}_{t}^{\dagger}\left(\mathbf{I}-\mathbf{S}_{a}^{\dagger} \mathbf{S}_{a}\right) \mathbf{T}_{t} \bar{a}_{s}}{\bar{a}_{s}^{\dagger} \mathbf{T}_{m}^{\dagger}\left(\mathbf{I}-\boldsymbol{\Gamma}_{s} \boldsymbol{\Gamma}_{s}^{\dagger}\right) \mathbf{T}_{m} \bar{a}_{s}}
$$

A few points about (11) merit further attention. First of all, note that in the transmission mode and in many arrays, there might be more than one excited port. The multi-port matching efficiency can still be achieved by virtue of the given equation. In this particular case, the foregoing efficiency metric may also be referred to as active matching efficiency [5]. In contrast, in diversity antenna systems, which are normally used in the receive mode, only the embedded element efficiency associated with each port is of concern. For this specific case, a single-entry (or single non-zero entry) excitation vector yields the associated multi-port matching efficiency. As long as the structure is lossless, the latter equates the associated total embedded element efficiency. It is interesting to also note that single-entry excitation vectors select out the diagonal entries in the expressions between $\bar{a}_{s}^{\dagger}$ and $\bar{a}_{s}$ in the numerator and denominator of (11). The off-diagonal entries in $\mathbf{T}_{t}^{\dagger}\left(\mathbf{I}-\mathbf{S}_{a}^{\dagger} \mathbf{S}_{a}\right) \mathbf{T}_{t}$ are the correlations between the ports of this system, which when normalized properly yield 
the corresponding correlation coefficients [9]. The absolute values of these correlation coefficients are similar to those of the random received signals at the corresponding ports in uncorrelated isotropic multipath environments if the system is lossless and we have a single-mode multi-port antenna [10].

Furthermore, in case only one cascaded network is present, the derived formula can still be simply adjusted to hold. To this end, just recall that the influence of each cascaded network can be removed by replacing $\mathbf{S}_{11}^{x, y}=\mathbf{S}_{22}^{x, y}=\mathbf{0}$ and $\mathbf{S}_{12}^{x, y}=\mathbf{S}_{21}^{x, y}=$ I. If both networks are to be removed, the given formula in (8) resembles the one in [5, eq. 3].

In principle, after implementation of an antenna system, there is little to do to reduce the losses inherent in it. Therefore, a key-role for the cascaded networks is to optimize the multiport matching efficiencies. This, in turn, will eventually lead to enhanced total embedded element efficiencies. Different possibilities for these desired matching networks have been discussed in the literature (for instance see [6]).

Finally, it is worth mentioning that the mean matching efficiency, which is a geometric mean value of all multi-port matching efficiencies associated with single-entry excitations, can be directly obtained from (11) [5].

\section{Formulation FOR N-CASCADED NETwORKS}

The approach pursued in the preceding section can readily be extended to $N$ cascaded networks. Let us consider a cut of three cascaded networks out of $N$. A schematic of these networks are shown in Fig. 2. Let us further index these networks as $(l-1), l$, and $(l+1)$, numbered respectively from the source side to the antenna side. The remainders of parameters to be used for our purpose are illustrated in the figure. As a point of caution, note that the indices used for the incident and reflected waves are different from those in Fig. 1. Recall also that the transmission matrix of the middle network, $\mathbf{T}_{l}$, links the two successive incident waves; that is, $\bar{a}_{l+1}=\mathbf{T}_{l} \bar{a}_{l}$. Using the network theory, it is quite straightforward to show that this transmission matrix is

$$
\mathbf{T}_{l}=\left(\mathbf{I}-\mathbf{S}_{22}^{l} \boldsymbol{\Gamma}_{l+1}\right)^{-1} \mathbf{S}_{21}^{l}
$$

which indicates that the transmission matrix at each network depends only on its scattering parameters as well as the reflection matrix at the input ports of the next cascaded network [11]. To achieve the transmission matrix of the next or preceding network, it is enough to replace $l$ with $(l \pm 1)$ in (12), respectively. Since the $N$-th cascaded network is connected to the antenna system with a known reflection matrix, i.e., $\boldsymbol{\Gamma}_{N+1}=\mathbf{S}_{a}$, the transmission matrix for the last network, $\mathbf{T}_{N}$, can be achieved by (12). Afterwards, to calculate the transmission matrix for $(N-1)$-th network, we need the reflection matrix at the input of the $N$-th network. To achieve this, following the same path as in (1), we can simply use

$$
\boldsymbol{\Gamma}_{l}=\mathbf{S}_{11}^{l}+\mathbf{S}_{12}^{l} \boldsymbol{\Gamma}_{l+1} \mathbf{T}_{l} .
$$

Having $\mathbf{S}^{l-1}$ and $\boldsymbol{\Gamma}_{l}$, we can continue to calculate the $\mathbf{T}_{l-1}$. This iterative process can be followed to eventually yield all
$\mathbf{T}_{N}, \mathbf{T}_{N-1}, \ldots, \mathbf{T}_{1}$. Now the total transmission matrix of the complete setup, which reads $\bar{a}_{a}=\mathbf{T}_{t} \bar{a}_{s}$, is given by

$$
\mathbf{T}_{t}=\mathbf{T}_{N} \cdots \mathbf{T}_{2} \mathbf{T}_{1} \mathbf{T}_{s}
$$

wherein $\mathbf{T}_{s}$ is defined in (2). As a point of caution, note that the maximum available power from a source depends on the source impedances and the source's power. Hence, it should be clear that the number of cascaded networks and their characteristics have no effect on $P_{\text {avs }}$ in (10). Ultimately, using (14), (10), and the general expression in (11) gives the multiport matching efficiency at the input of $N$-cascaded networks connected to an arbitrary multi-port antenna.

\section{Formulation of DeCOUPLing EFFICIENCY}

Decoupling efficiency is defined as the ratio between the accepted power and the incident power at the ports of a multiport antenna system [4]. The main difference between multiport matching efficiency and decoupling efficiency is that the former includes the effects of source impedances in total radiation performance of a multi-port antenna, whereas the latter does not. For this reason, the multi-port matching efficiency is of more practical interest than its counterpart. Despite this, based on the analysis presented in the preceding sections, we are able to form a general expression for calculation of decoupling efficiency in the presence of $N$-cascaded networks. Referring back to Fig. 1, the total incident power to the input of cascaded networks, $P_{\text {inc }}$, is

$$
P_{\text {inc }}=\bar{a}_{x}^{\dagger} \bar{a}_{x}=\bar{a}_{s}^{\dagger} \mathbf{T}_{s}^{\dagger} \mathbf{T}_{s} \bar{a}_{s}
$$

The accepted power is obtained by (8), wherein $\mathbf{T}_{t}$ for the general case of $N$-cascaded networks is given in (14). Now, the decoupling efficiency, $e_{\mathrm{dcl}}$, associated with a certain excitation scheme, $\bar{a}_{s}$, can be achieved by

$$
\left.e_{\mathrm{dcl}}\right|_{\bar{a}_{s}}=\frac{\bar{a}_{s}^{\dagger} \mathbf{T}_{t}^{\dagger}\left(\mathbf{I}-\mathbf{S}_{a}^{\dagger} \mathbf{S}_{a}\right) \mathbf{T}_{t} \bar{a}_{s}}{\bar{a}_{s}^{\dagger} \mathbf{T}_{s}^{\dagger} \mathbf{T}_{s} \bar{a}_{s}}
$$

In general, the decoupling efficiency takes into account the effects of reflections and coupling between different elements. In contrast, as mentioned before, the multi-port matching efficiency, not only includes these, but also contains the effects of terminating impedances in the overall radiation performance of the system. Nevertheless, an interesting case can be thought of wherein source impedances are matched to the characteristic impedances of the multi-port system; that is, $\boldsymbol{\Gamma}_{s}=\mathbf{0}$. Under this constraint and after some manipulations, we have

$$
\mathbf{T}_{m}\left(\boldsymbol{\Gamma}_{s}=\mathbf{0}\right)=\mathbf{T}_{s}\left(\boldsymbol{\Gamma}_{s}=\mathbf{0}\right)=0.5 \mathbf{I}
$$

The above expression demonstrates that the decoupling efficiency in (16) equates the multi-port matching efficiency in (11) if the source impedances are matched to the characteristic impedances of the multi-port system. 


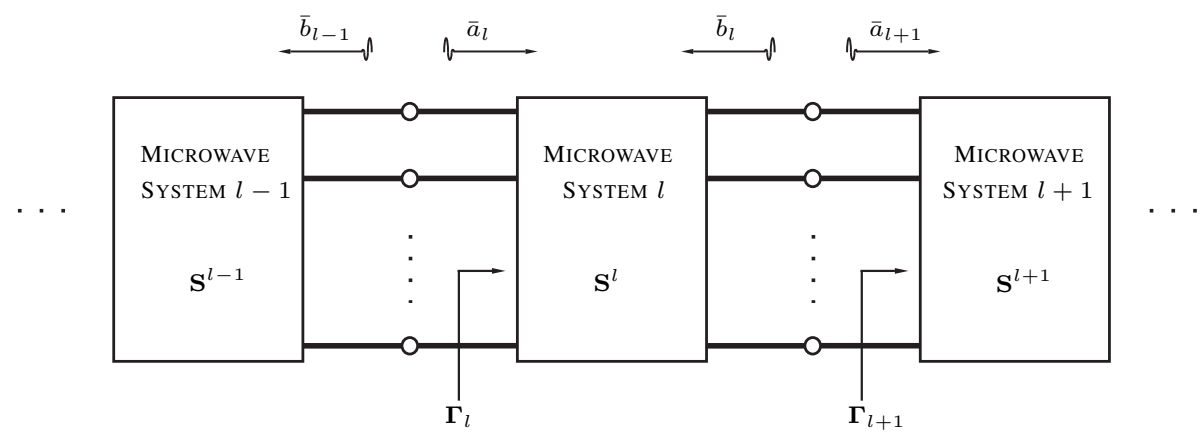

Fig. 2. Microwave circuit model of three consecutive cascaded networks.

\section{CONCLUSION}

In this paper, a compact formula is provided for calculation of multi-port matching efficiency in the presence of two cascaded passive microwave networks. Moreover, the compact formula is extended to include the cases of arbitrary number of cascaded networks. In case of a lossless system and singlemode multi-port antennas, an expression for covariance matrix of the complete setup is provided, which is valid in rich uncorrelated isotropic multipath environments. A comparison between the decoupling efficiency and the multi-port matching efficiency is also presented. The elaborated analysis is used to yield a compact formula for the decoupling efficiency too. It is shown that under matched termination constraint, the two aforementioned efficiency metrics become identical. Using the presented material, the mean matching efficiency, which is used for a quick estimation of diversity gain, is addressed. Note that, all derived compact formulas can be advantageous for engineers whose aim is to optimize the radiation performance of a multi-port antenna system by designing a suitable multiport matching network.

\section{REFERENCES}

[1] M. Manteghi and Y. Rahmat-Samii, "Multiport characteristics of a wide-band cavity backed annular patch antenna for multipolarization operations," IEEE Transactions on Antennas and Propagation, vol. 53, no. 1, pp. 466-474, January 2005.

[2] T. Taga, "Analysis for mean effective gain of mobile antennas in land mobile radio environments," IEEE Transactions on Vehicular Technology, vol. 39, no. 2, pp. 117-131, May 1990.

[3] K. Rosengren and P.-S. Kildal, "Radiation efficiency, correlation, diversity gain and capacity of six monopole antenna array for a MIMO system: Theory, simulation and measurement in reverberation chamber," Proceedings IEE, Microwaves Antennas and Propagation, 2005.

[4] M. Ivashina, M. Kehn, P.-S. Kildal, and R. Maaskant, "Decoupling efficiency of a wideband vivaldi focal plane array feeding a reflector antenna," IEEE Transactions on Antennas and Propagation, vol. 57, no. 2, pp. 373-382, February 2009.

[5] N. Jamaly and A. Derneryd, "Efficiency characterization of multi-port antennas," Electronics Letters, vol. 48, no. 4, February 2012.

[6] B. K. Lau, J. Andersen, G. Kristensson, and A. Molisch, "Impact of matching network on bandwidth of compact antenna arrays," IEEE Transactions on Antennas and Propagation, vol. 54, no. 11, pp. 32253238, November 2006.

[7] D. M. Pozar, Microwave Engineering, 3rd ed. John Wiley \& Sons, 2005.

[8] W. Wiesbeck and E. Heidrich, "Wide-band multiport antenna characterization by polarimetric RCS measurements," IEEE Transactions on Antennas and Propagation, vol. 46, no. 3, pp. 341-350, March 1998.
[9] J. W. Wallace and M. A. Jensen, "Termination-dependent diversity performance of coupled antennas: Network theory analysis," IEEE Transactions on Antennas and Propagation, vol. 52, no. 1, pp. 98-105, January 2004.

[10] R. Vaughan and J. Andersen, "Antenna diversity in mobile communications," IEEE Transactions on Vehicular Technology, vol. 36, no. 4, pp. 149-172, November 1987.

[11] R. E. Collin, Foundations for Microwave Engineering, 2nd ed. IEEE Press, 2001. 